Open Access

\title{
Wideband partial response CPM demodulation via multirate frequency transformations and decision feedback equalization
}

\author{
Wenjing Liu* (D) and Balu Santhanam
}

\begin{abstract}
Continuous phase modulation (CPM) is a popular frequency modulation technique used in mobile communications due to its power efficiency and constant modulus properties. Conventional narrowband CPM demodulation employs the Viterbi algorithm after phase demodulation and requires that the phase states be rational and contain additive white noise. The complexity of the Viterbi approach further increases with the number of phase states. Frequency discrimination approaches that estimate the instantaneous frequency provide a simpler suboptimal approach but are primarily for full response CPM and are not well known for wideband partial response CPM.

In this paper, we investigate an approach that combines multirate frequency transformations for wideband CPM demodulation with decision feedback equalization for memory removal. This combined approach avoids the problems of complexity and restrictive requirements of the Viterbi approach. Simulation results are used to demonstrate the validity of the combined approach.
\end{abstract}

Keywords: AM-FM demodulation, Continuous phase modulation, Multirate frequency transformations, Energy separation algorithm, Decision feedback equalization

\section{Introduction}

Continuous phase modulation (CPM) [1-3] is a popular form of frequency modulation employed in mobile communications [4] and has desirable spectral efficiency [5] and constant modulus properties that facilitate use of class-C amplifiers. Gaussian Minimum Shift Keying (GMSK), a specific form of CPM, is the main ingredient in the Global System for Mobile communications (GSM) $[2,3]$ used in GPS applications. Pragmatic CPM modulation schemes have recently been studied as capacity attaining low-complexity alternatives to serially concatenated CPM [6].

The conventional demodulation technique used for narrowband signals is phase demodulation [7] followed by unwrapping and maximum likelihood sequence estimation (MLSE) using the Viterbi algorithm [2, 3, 8]. This

*Correspondence: wenjingliu1989@gmail.com

Department of Electrical and Computer Engineering, University of New Mexico, Albuquerque, NM, 87106, USA approach has a complexity that grows exponentially with the number of phase states and restrictions on the modulation index that needs to be the ratio of incommensurate integers. Other frequency discrimination approaches [9] rely upon instantaneous frequency (IF) estimation and are not subject to the restrictions required by the Viterbi but have not been investigated for wideband CPM with memory.

In prior work [10, 11], it was shown that frequency discrimination for full response CPM demodulation has the same performance as that of binary phase-shift keying (BPSK) detection in additive white Guassian noise (AWGN). Further, in recent work [12], frequency tracking-based wideband FM demodulation was extended to large wideband to narrowband conversion factors using multirate frequency transformations (MFT). Frequency estimation-based approaches have the added advantage that they are immune to phase distortions introduced by the channel which would adversely affect the Viterbi 
approach. In addition, these approaches do not require prior knowledge of the carrier frequency.

In this paper, we investigate the approach that combines the MFT and energy separation algorithm (ESA) [13, 14] with decision feedback equalization (DFE) to effectively demodulate wideband CPM with memory. The wideband CPM signal is first converted into narrowband via the MFT and then demodulated by the ESA to obtain the IF estimates. The DFE is eventually applied to equalize the partial response channel generated from the IF estimates in order to remove the CPM memory introduced by partial response signaling. Simulation results are used to verify the efficacy of the combined approach for both wideband binary CPM and multilevel CPM.

\section{Wideband CPM demodulation}

\subsection{AM-FM signal model}

Monocomponent amplitude-modulation frequencymodulation (AM-FM) signals are expressed in the form of time-varying sinusoids by

$$
s(t)=a(t) \cos \left(\int_{-\infty}^{t} \omega_{i}(\tau) d \tau+\theta_{1}\right)
$$

where the instantaneous amplitude (IA) is denoted by $a(t)$ and the instantaneous frequency (IF) is given by

$$
\omega_{i}(t)=\omega_{c}+\omega_{m} q_{i}(t) .
$$

Note that $\omega_{c}$ is the carrier (or mean) frequency, and $q_{i}(t)$ is the normalized baseband-modulated signal.

Specific for sinusoidal FM, where $a(t)$ remains a constant $A$, and $q_{i}(t)$ becomes a sinusoid, the IF can be further expressed as

$$
\omega_{i}(t)=\omega_{c}+\omega_{m} \cos \left(\omega_{f} t+\theta_{2}\right) .
$$

Sinusoidal FM signals can be expressed via:

$$
s(t)=A \sum_{n=-\infty}^{+\infty} J_{n}(\beta) \cos \left(\omega_{c} t+n \omega_{m} t\right),
$$

where $J_{n}$ is the $n$th order cylindrical Bessel function of the first kind. The modulation index of the sinusoidal FM is defined as the ratio $\beta=\omega_{m} / \omega_{f}$ and the associated Carson bandwidth is given by

$$
B=2(\beta+1) \omega_{f} \text {. }
$$

If $\beta \gg 1$, then it corresponds to the wideband FM according to the literature of FM communication systems. In addition, the carrier-to-information-bandwidth ratio $(\mathrm{CR} / \mathrm{IB})$ and the carrier-to-frequency-deviation ratio $(\mathrm{CR} / \mathrm{FD})$ are defined respectively as:

$$
\frac{\mathrm{CR}}{\mathrm{IB}}=\frac{\omega_{c}}{\omega_{f}}, \frac{\mathrm{CR}}{\mathrm{FD}}=\frac{\omega_{c}}{\omega_{m}} .
$$

Wideband FM is a popular modulation technique for satellite communications due to its ability to deal with trans-ionospheric distortion. A particular form of digital FM, multilevel Gaussian fequency-shift keying (FSK) has been proposed as a solution for high bandwidth satellite communications [15-17].

\subsection{Continuous phase modulation}

CPM can be viewed as a specific form of FM. The standard CPM model depends on its pulse shaping function $p(t)$, with duration length of symbol periods $L$ and modulating symbols, i.e, binary PAM symbols $a[k] \in\{-1,1\}$. The IF signal takes the form $[2,3]$ :

$$
\omega_{i}(t)=\omega_{c}+2 \pi h \sum_{k=-\infty}^{\infty} a[k] p\left(t-k T_{b}\right),
$$

where $\omega_{c}$ is the carrier frequency and $h$ is the modulation index of CPM. Note that in the Viterbi algorithm, the modulation index must be rational, which is restricted to be of the form:

$$
h=\frac{m}{p}
$$

where $m$ and $p$ are relatively prime positive integers. The deviation of the phase from the carrier phase is given by:

$$
\phi_{\mathrm{dev}}(t ; \mathbf{a})=2 \pi h \sum_{k=-\infty}^{\infty} a[k] q\left(t-k T_{b}\right),
$$

where $p(t)$ denotes the pulse shaping function, which is usually normalized and defined in the interval $\left[0, L T_{b}\right]$, and $q(t)$ is the corresponding phase pulse shaping function defined by

$$
q(t)=\int_{0}^{t} p(\tau) d \tau
$$

In general, the following conditions are satisfied by the choice of $p(t)$ :

$$
\begin{aligned}
& p(t)=p\left(L T_{b}-t\right), \\
& q(t)=\int_{0}^{t} p(\tau) d \tau=\frac{1}{2}, \quad t \geq L T_{b} .
\end{aligned}
$$

If $p(t)$ is a rectangular pulse, then this form of CPM is referred to as (L-REC) CPM, and if $p(t)$ is a raised cosine pulse, then it is referred to as (L-RAC) CPM. The CPM signal is then obtained via frequency modulation:

$$
r(t)=A \cos \left(\int_{-\infty}^{t} \omega_{i}(\tau) d \tau+\theta\right) .
$$

Usually, the modulation index of a wideband CPM signal is large such that the frequency deviation of its IF is comparable to the carrier frequency.

Using a pulse shaping function of duration larger than a symbol period $(L>1)$, i.e., partial response signaling introduces memory into the modulation scheme, but 
results in significant increase of complexity for demodulation and detection. The memory introduced by CPM depends on the type of its pulse shaping function $p(t)$ and the corresponding duration length $L$. Since $p(t)$ is defined over the interval $\left[0, L T_{b}\right]$, for $n T_{b}<t \leq(n+1) T_{b}$, Eq. 7 can be rewritten as

$$
\omega_{i}(t)=\omega_{c}+2 \pi h \sum_{k=n-L+1}^{n} a[k] p\left(t-k T_{b}\right) .
$$

Given the carrier frequency and modulation index, the information conveyed in the IF within the current symbol period depends on the most recent $L$ symbols and the waveform of $p(t)$.

\subsection{Energy separation algorithm}

The energy separation algorithm (ESA) as summarized in $[13,14]$, based on the Teager-Kaiser energy operator $\Psi[x(t)]=\dot{x}^{2}(t)-x(t) \ddot{x}(t)$, is widely used for monocomponent AM-FM demodulation, for example, to analyze the oscillation of signals with time-varying amplitude and frequency. The IA $a(t)$ and the IF $\omega_{i}(t)$ of an AM-FM signal $x(t)$ can be estimated via the continuous ESA (CESA) summarized by

$$
\begin{aligned}
& \frac{\Psi[x(t)]}{\sqrt{\Psi[\dot{x}(t)]}} \approx|a(t)| . \\
& \sqrt{\frac{\Psi[\dot{x}(t)]}{\Psi[x(t)]}}-\omega_{c} \approx \omega_{i}(t),
\end{aligned}
$$

where we assume that the IA $a(t)$ and the IF $\omega_{i}(t)$ do not vary too fast or too greatly in value compared to the carrier frequency $\omega_{c}$.

\subsection{Carrier frequency and amplitude estimation}

The IF estimate of the MFT-ESA in the specific case of CPM takes the form of:

$$
\hat{\omega}_{i}(t)=\omega_{c}+2 \pi h \sum_{k=-\infty}^{\infty} a[k] h_{f}\left(t-k T_{b}\right)+\epsilon_{\omega}(t),
$$

where $h_{f}(t)$ corresponds to the pulse shaping function and $\epsilon_{w}(t)$ corresponds to zero-mean IF noise, which unlike the observation noise is not white. Assuming equiprobable symbols and taking expectations on both sides yields:

$$
E\left\{\hat{\omega}_{i}(t)\right\}=\omega_{c} .
$$

The carrier frequency and the amplitude of the AM-FM signal can then be estimated from the IF and IA estimates from either algorithm by simple averaging:

$$
\begin{gathered}
\hat{\omega}_{c}=\frac{1}{T} \int_{0}^{T} \hat{\omega}_{i}(t) d t \\
\hat{A}=\frac{1}{T} \int_{0}^{T} \hat{a}_{i}(t) d t .
\end{gathered}
$$

This is a consequence of the fact that these approaches are bandpass estimation approaches whereas traditional in-phase and quadrature demodulation, employed in narrowband communication systems, is a baseband estimation approach requiring prior knowledge of the carrier frequency.

\subsection{Multirate frequency transformations}

The performance of ESA or any other demodulation technique directly applied to wideband FM or CPM signals is poor due to the narrowband constraint, as demonstrated in prior work [12]. In recent work of the authors, frequency transformations enacted via multirate signal processing as shown in Fig. 1 were used for wideband FM to narrowband FM conversion to enable a wider range of wideband FM signals $[10,12]$ and were also extended to two-dimensional images [18] via multidimensional energy operator [19]. The goal of the multirate processing module is to compress the bandwidth of the FM signal; however, this is accompanied by a reduction in the carrier frequency of the FM signal. To compensate, a heterodyning module that translates the FM signal in frequency is introduced. After the multirate heterodyne combination, the $\mathrm{CR} / \mathrm{IB}$ and $\mathrm{CR} / \mathrm{FD}$ of the transformed signal is constrained in a range, where standard narrowband monocomponent FM demodulation algorithms work optimally. The MFT framework can be combined with a variety of demodulation techniques such as the Hilbert transform demodulation algorithm (HTDA) [20] or the ESA to improve the demodulation perforamnce for wideband FM signals. In particular, the ESA combined with the MFT approach will be employed in this paper.

The MFT framework specifically allows for demodulation of wideband CPM signals with a large modulation depth as the examples shown in subsequent sections. Since the approach is based on IF estimation, the approach does not encounter the complexity problems or restrictions related to rational modulation depth as seen in the Viterbi algorithm.

\section{Memory removal of partial response CPM}

\subsection{Partial response channel}

The memory introduced by the partial response signaling linearly distorts the transmitted signal and results in the intersymbol interference (ISI) for the IF of the CPM signal within each symbol period. If precise IF estimates of the CPM signal are accessible, the recovery of the original transmitted sequence is similar to equalization of the ISI channel.

A discrete time-invariant channel is generally expressed as

$$
y(t)=\sum_{m=-\infty}^{\infty} h_{m} x(t-m)+n(t) .
$$




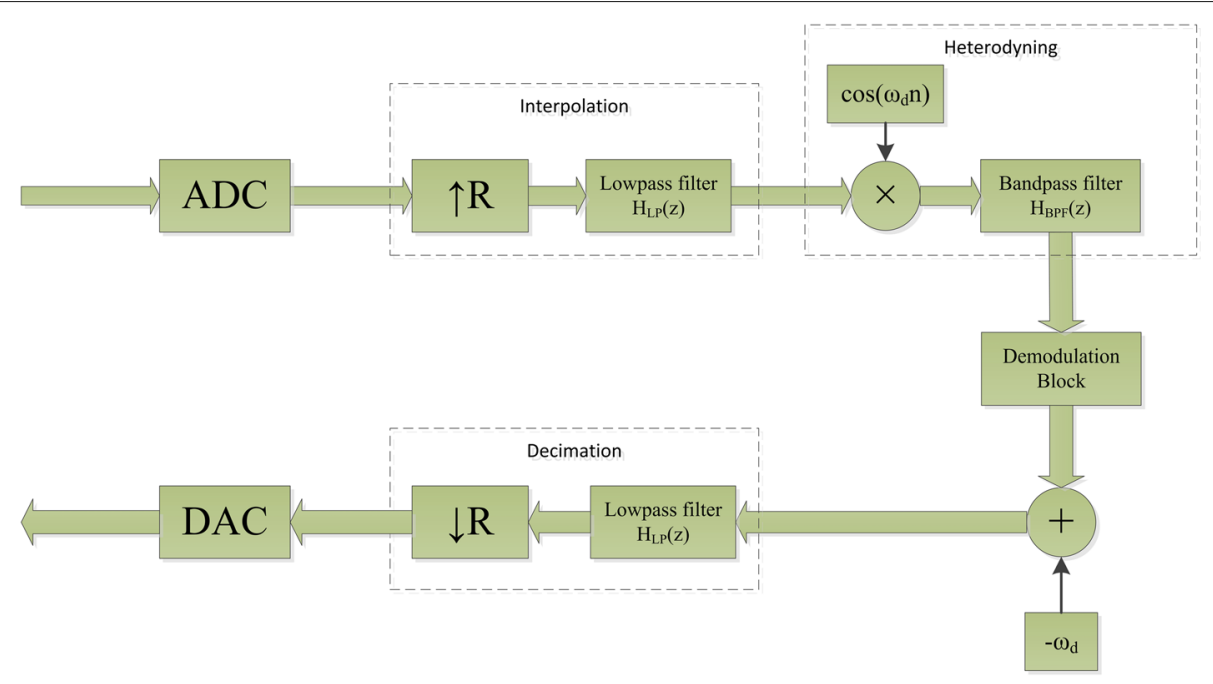

Fig. 1 Block diagram of the basic MFT framework. The wideband signal is first sampled above the Nyquist rate, interpolated by a factor $R$, and then heterodyned by multiplication with $\cos \left(\omega_{d} n\right)$, followed by a discrete FIR bandpass filter with a passband gain to achieve the MFT. Then, it goes through a demodulation block to generate IF estimates of the compressed heterodyned signal. To obtain the IF of the original signal, the compressed heterodyned IF is then shifted back by subtracting $\omega_{d}$, decimated by $R$, and scaled back appropriately, followed by the DAC module

where $h_{m}$ is the impulse response, $x(t)$ is the input sequence of the channel, and $n(t)$ is the noise of the channel.

In terms of the normalized IF within each symbol period, the partial response channel is a special case of the ISI channel that has a finite-length impulse response and is also causal and monic with $h_{0}=1$, according to Eq. 14 . For example, the discrete-time partial response channel using L-REC CPM can be modeled as

$$
m(t)=\sum_{k=0}^{L-1} s(t-k)+z(t),
$$

where $s(t)$ is the original transmitted symbol sequence and $z(t)$ is the noise term averaged from the noise of the IF estimates within each symbol period. Unlike the baseband channel assuming additive white Guassian noise (AWGN), the noise term $z(t)$ for this partial response channel of CPM is not AWGN since the noise presented in the IF estimates cannot be guaranteed as AWGN by any IF demodulation approach.

The output sequence $m(t)$ of the partial response channel can be obtained by demodulating the CPM signals and making decisions on the normalized demodulated IF within each symbol period. Therefore, our goal becomes recovering the original transmitted sequence from the output of the partial response channel.

\subsection{Partial response channel estimation via recursive prediction error method}

The channel response is required by general equalization approaches, in order to remove the memory of the partial response CPM. In this paper, we perform the channel estimation via the recursive prediction error method based on the ARMAX model as described in [21]. The partial response channel, depicted for example in Eq. (21), is actually a monic moving-average (MA) process. We are capable of estimating the channel response by fitting the output of the partial response channel to an ARMAX model.

In general, the structure of the ARMAX model is described by

$$
\sum_{k=0}^{n_{a}} a_{k} y[t-k]=\sum_{k=1}^{n_{b}} b_{k} u[t-k]+\sum_{k=0}^{n_{c}} c_{k} e[t-k],
$$

where $n_{a}, n_{b}$, and $n_{c}$ are the number of coefficients for the auto-regressive (AR) part, system input, and movingaverage (MA) part, respectively. It can also be written as

$$
A(q) y(t)=B(q) u(t)+C(q) e(t)
$$

where $q$ is the backward shift operator. Specifically,

$$
\begin{aligned}
& A(q)=1+a_{1} q^{-1}+\ldots+a_{n_{a}} q^{-n_{a}}, \\
& B(q)=b_{1} q^{-1}+\ldots+b_{n_{b}} q^{-n_{b}} . \\
& C(q)=1+c_{1} q^{-1}+\ldots+c_{n_{b}} q^{-n_{c}} .
\end{aligned}
$$

By assuming $A(q)=1$ and $B(q)=0$, the ARMAX model can be simplified to the MA model that exactly fits Eq. (21), as described by

$$
y(t)=C(q) e(t)=\sum_{k=0}^{n_{c}} c_{k} q^{-k} e(t)=\sum_{k=0}^{n_{c}} c_{k} e[t-k] .
$$


By satisfying certain conditions, $C(q)$ is actually invertible, that is, $e(t)$ can be calculated via an inverse operator $\tilde{C}(q)$ via

$$
e(t)=\tilde{C}(q) y(t)=\sum_{k=0}^{\infty} \tilde{c}_{k} y(t-k) .
$$

The coefficient estimation for the ARMAX model can be achieved via an iterative search algorithm that minimizes a more robust quadratic prediction error criterion. The parameter vector $\vec{\theta}$ can be formed by grouping the coefficients of the ARMAX model as

$$
\vec{\theta}=\left[a_{1}, \ldots, a_{n_{a}}, b_{1}, \ldots, b_{n_{b}}, c_{1}, \ldots, c_{n_{c}}\right] .
$$

The predictor for the ARMAX model is given by

$$
C(q) \hat{y}(t \mid \vec{\theta})=B(q) u(t)+[C(q)-A(q)] y(t) .
$$

It can be rewritten as

$$
\hat{y}(t \mid \vec{\theta})=B(q) u(t)+[1-A(q)] y(t)+[C(q)-1] \epsilon(t, \vec{\theta}),
$$

where $\epsilon(t, \vec{\theta})$ is defined as the prediction error given by

$$
\epsilon(t, \vec{\theta})=y(t)-\hat{y}(t \mid \vec{\theta}) .
$$

Therefore, we can express the predictor in the form of pseudolinear regression via

$$
\hat{y}(t \mid \vec{\theta})=\vec{\varphi}^{T}(t, \vec{\theta}) \vec{\theta},
$$

where we define the data vector $\vec{\varphi}(t, \vec{\theta})$ as

$$
\begin{aligned}
\vec{\varphi}(t, \vec{\theta})= & {\left[-y(t-1), \ldots,-y\left(t-n_{a}\right), u(t-1), \ldots,\right.} \\
& \left.u\left(t-n_{b}\right), \epsilon(t-1, \vec{\theta}), \ldots, \epsilon\left(t-n_{c}, \vec{\theta}\right)\right] .
\end{aligned}
$$

According to Eq. (31), the gradient of the predictor $\vec{\psi}(t, \vec{\theta})$ w.r.t $\vec{\theta}$ can be computed via

$$
C(q) \vec{\psi}(t, \vec{\theta})=\vec{\varphi}(t, \vec{\theta}) .
$$

The gradient $\vec{\psi}(t, \vec{\theta})$ can be obtained by filtering the data vector $\vec{\varphi}(t, \vec{\theta})$ through an inverse filter of $C(q)$.
The cost function for the recursive prediction error method is defined as

$$
V_{t}\left(\vec{\theta}, \vec{Y}^{t}\right)=\gamma(t) \frac{1}{2} \sum_{k=1}^{t} \beta(t, k) \epsilon^{2}(k, \vec{\theta}),
$$

where $\beta(t, k)$ and $\gamma(t)$ satisfy the following conditions

$$
\begin{aligned}
& \beta(t, k)=\prod_{j=k+1}^{t} \lambda(j), \quad \beta(t, t)=1, \\
& \sum_{k=1}^{t} \gamma(t) \beta(t, k)=1,
\end{aligned}
$$

Note that $\lambda(j)$ is the forgetting factor, which is often set to a constant less than 1 . It controls the convergence rate and leads to compromise between misadjustment and tracking, similar to the recursive least squares (RLS) approach in the context of adaptive filtering [22].

The algorithm of the recursive prediction error method is then summarized via

$$
\begin{aligned}
& \epsilon(t)=y(t)-\hat{y}(t), \\
& \hat{\vec{\theta}}(t)=\hat{\vec{\theta}}(t-1)+\gamma(t) R^{-1}(t) \vec{\psi}(t) \epsilon(t), \\
& R(t)=R(t-1)+\gamma(t)\left[\vec{\psi}(t) \vec{\psi}^{T}(t)-R(t-1)\right],
\end{aligned}
$$

where $\vec{\psi}(t)$ and $\hat{y}(t)$ are short for the resulting approximations of $\vec{\psi}(t, \hat{\vec{\theta}}(t-1))$ and $\hat{y}(t \mid \vec{\theta}(t-1))$, respectively.

\subsection{Memory removal via decision feedback equalization} Similar to eliminating ISI, decision feedback equalization (DFE) [23] can be applied to the output sequence of the partial response channel to remove the CPM memory. The block diagram of the general decision feedback equalizer is illustrated in Fig. 2. According to the choice of the feedforward filter and feedback filter, a variety of decision feedback equalizers can be implemented, such as the

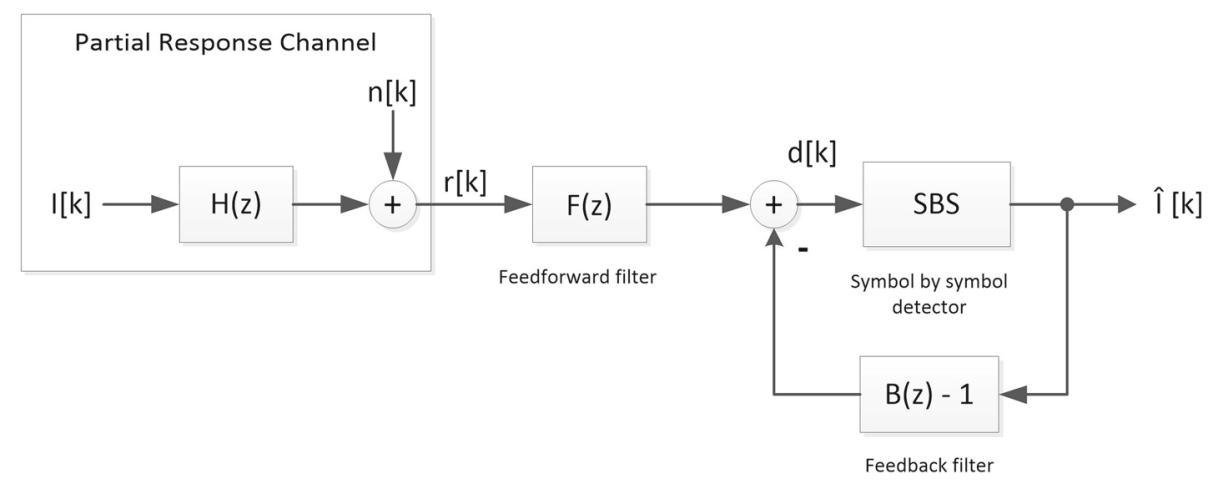

Fig. 2 Block diagram of the general decision feedback equalizer. The partial response channel output in this case are the mixed symbols due to memory introduced by partial response signaling. The mixed symbols can be obtained from the estimated IF of the MFT-ESA demodulation block 
zero-forcing DFE that directly inverts the channel and the MMSE-DFE that employs a minimum mean-square error criterion.

\subsubsection{ZF-DFE solution}

By inverting the partial response channel using the estimated channel response, the original symbol sequence can be recovered by applying the inverse filter $H_{\text {inv }}(q)$ on the channel output sequence $m(t)$ and classifying the filter output according to the optimal region of each symbol. For the previous case of the 3-REC multilevel CPM, the expression for the inverse filter is given by

$$
H_{\text {inv }}(q)=\frac{1}{1+\hat{c}_{1} q^{-1}+\hat{c}_{2} q^{-2}} .
$$

Note that the inverse filter $H_{\text {inv }}(q)$ is an IIR All-pole filter, which can be simply implemented via direct recursion of its difference equation by

$$
\hat{s}(t)=m(t)-\hat{c}_{1} \hat{s}(t-1)-\hat{c}_{2} \hat{s}(t-2),
$$

where $\hat{s}$ represents the estimated symbol sequence. It requires the input, i.e., in our case, the partial response channel output sequence $m(t)$, to be nearly perfect; otherwise, the error incurred by inaccurate input symbols can be significant. This in turn requires the MFT-ESA demodulation section to produce a sufficiently accurate demodulation result. By incorporating the slicer, i.e., the symbol by symbol detection device into the inverse filter recursion, we can implement the decision feedback version of the zero-forcing equalizer (ZF-DFE) to eliminate memory induced in the partial response CPM signals.

\subsubsection{MMSE-DFE solution}

Instead of focusing on just removal of the channel between the IF input and the information symbols, if we further incorporate a MMSE cost function that balances the task of eliminating memory while simultaneously reducing symbol distortion, we obtain the linear MMSE equalizer that can provide further improvement in the symbol error performance in low signal-to-noise ratio (SNR) environment. The corresponding decision feedback version of the linear MMSE equalizer (MMSEDFE) incorporates both pre-cursor and post-cursor taps. The feedforward filter coefficients of the MMSE-DFE are obtained from the Wiener solution and then used to solve for the feedback filter coefficients. A detailed description of the MMSE-DFE solution is presented in [24].

\section{Simulation results}

\subsection{Performance of carrier frequency estimation}

In practice, since prior knowledge of distributions is not available, we replace the expectation with a simple timeaverage as in Eq. 19. While in the discrete-time case, we replace the integral with a time-average sum:

$$
\hat{\Omega}_{c}=\frac{1}{L} \sum_{k=0}^{L-1} \Omega_{i}[k] .
$$

Figure 3 depicts the carrier frequency estimation error of the MFT-ESA for the case where we have equiprobable symbols versus SNR. For larger SNR values, the carrier frequency estimation error approaches zero indicating that the IF yields a reliable carrier frequency estimate.

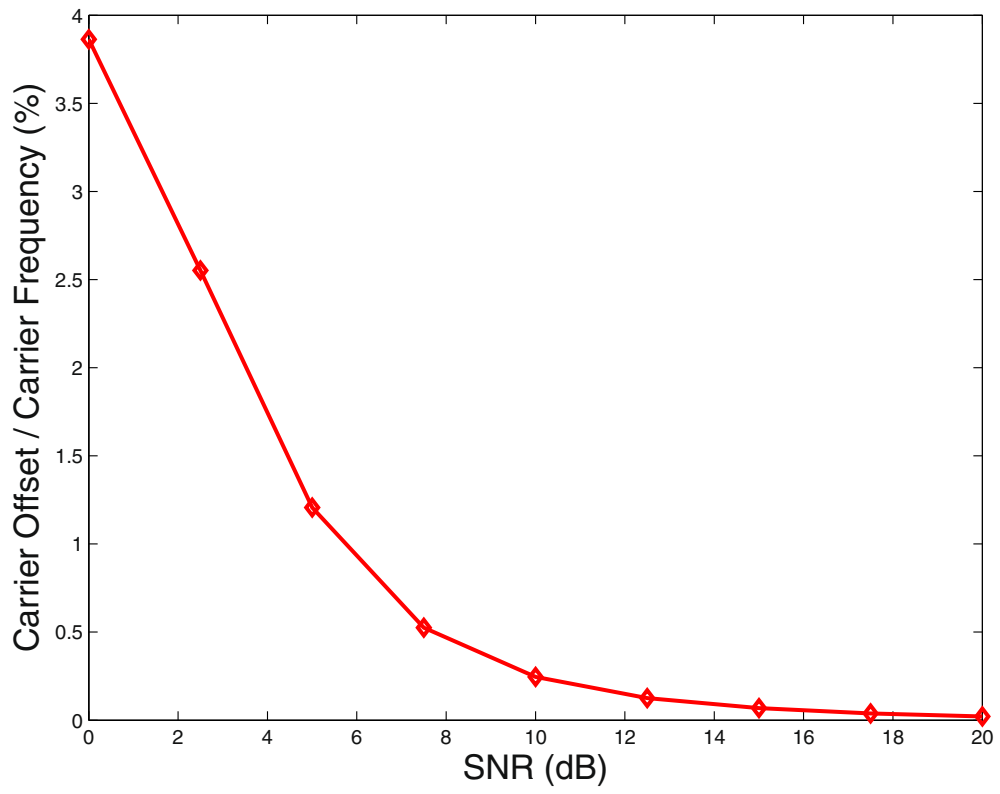

Fig. 3 Performance of carrier frequency estimation for the MFT-ESA approach 


\subsection{Performance of wideband partial response multilevel CPM demodulation}

A wideband 3-REC multilevel CPM signal with symbols taking values in the alphabet $\{-3,-1,1,3\}$ is used for performance test. The frequency deviation of the IF is equal to the carrier frequency in this extreme wideband case with modulation index $h=4$. The symbol error probability for the proposed MFT-ESA approach is depicted in Fig. 4. Since the 3-REC CPM signal has memory, the symbols here refer to the mixed symbols due to the memory effect of partial response signaling as described in Eq. 21. As the SNR increases, the MFT and ESA combination reduces the error dramatically. The error eventually drops to zero when the SNR passes certain threshold, while the error performances of the Hilbert transform (HTDA) and the ESA gradually saturate at certain levels due to carry-over effects from incomplete demodulation induced by narrowband constraints.

Unlike the common AWGN channel, the AWGN imposed on the CPM signal is not linearly added to the modulation signals (or symbols) in the partial response channel since the modulation signals (or symbols) are conveyed in the IF of the CPM signal. Therefore, the symbol error performance for the CPM signal is different from what is observed with modulation schemes that fit into the AWGN channel analysis, such as the classic Q curve for BPSK modulation. The CPM format provides robustness to noise when the SNR exceeds a certain threshold and if the CPM signal is sufficiently sampled.

\subsection{Performance of partial response channel estimation via recursive error method}

The simulation result of the partial response channel estimation via the recursive error method for wideband 3-REC multilevel CPM is illustrated by Fig. 5. With a memory length $L=3$, the partial response channel for the 3-REC multilevel CPM can be expressed via

$$
m[t]=s[t]+c_{1} s[t-1]+c_{2} s[t-2]+z(t),
$$

where $c_{1}=c_{2}=1$. As we can observe from Fig. 5 , the estimated coefficients converge close to the true value 1 in the case of REC CPM. They serve as useful estimates when other forms of pulse shaping such as in RAC-COM or SRAC-CPM are employed.

\subsection{Performance of partial response CPM memory removal via decision feedback equalization}

The MFT-ESA demodulation module described in the prior sections is then combined with the decisionfeedback equalization for memory removal to obtain estimates of the original information symbols. The channel response required by equalization has been estimated via the recursive error method.

The symbol error probability performance of the proposed MFT-ESA demodulation combined with ZF-DFE and MMSE-DFE for memory removal are compared in Fig. 6 for both wideband binary and multilevel 3-REC CPM senarios. Note that the modulation indices of the wideband CPM signals in this example are deliberately

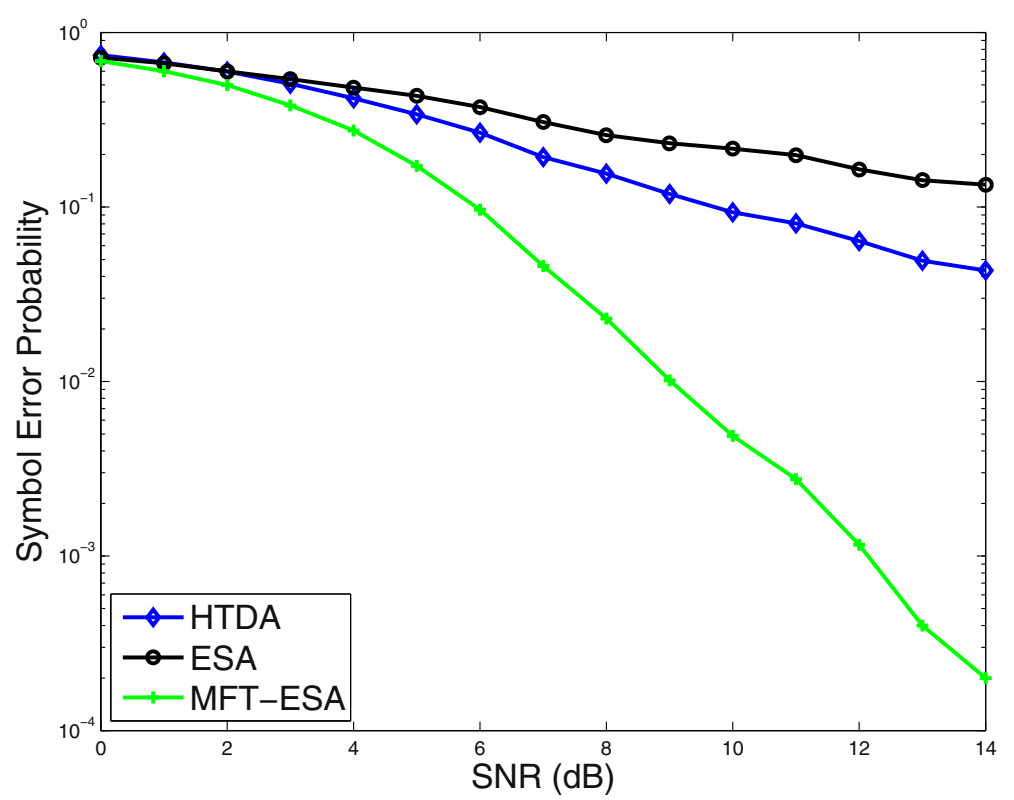

Fig. 4 Symbol error probability associated with the mixed symbols for wideband 3-REC multilevel CPM. Note that the SEP will drop to zero around $16 \mathrm{~dB}$ for the MFT-ESA approach, which is not shown due to limitations of the log scale 


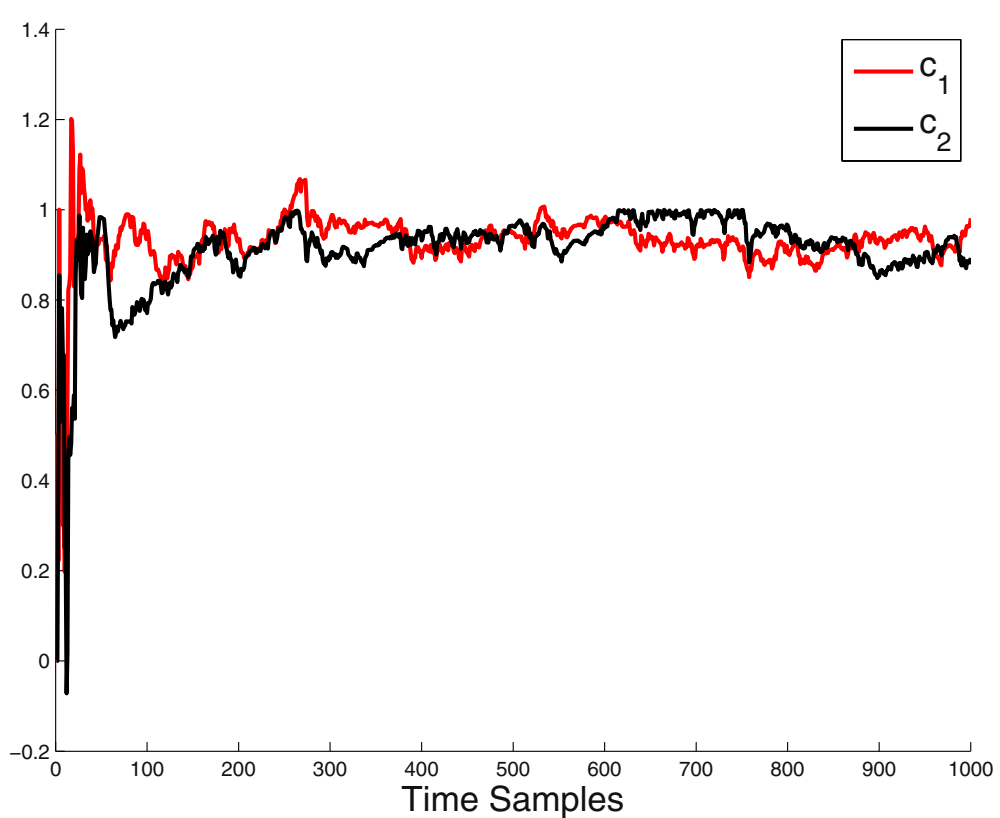

Fig. 5 Partial response channel estimation for 3-REC multilevel CPM via recursive error method. Note that the multilevel symbols take values in $\{-3,-1,1,3\}$. The red line indicates the convergence of the estimated coefficient $\hat{c}_{1}$, and the black line indicates the convergence of the estimated coefficient $\hat{c}_{2}$

chosen such that the implementation of the Viterbi algorithm is not practical due to the complexity of its phase states. For binary case, the MMSE-DFE performs slightly better than the zero-forcing DFE in the low SNR region, as shown in Fig. 6a. For multilevel case, the performance of both approaches are almost the same as in Fig. 6b. Since the multilevel signaling compress the decision region for symbol detection, the resolution of the symbol by symbol detector (or the slicer) is reduced, resulting in degraded performances of both approaches at the same level in the extreme wideband case where the IF deviation is close to the carrier frequency.

From our previous analysis, we know that the error associated with the mixed symbols (or output of the partial response channel) obtained from the demodulated IF for low SNR environment is significant due to error propagation. The recovery of the original symbols for low SNR environment is hence significantly influenced since the proposed memory removal approach is very sensitive to its input. However, as the SNR increases, the output of the partial response channel determined by MFT-ESA demodulation module becomes more accurate, leading to a significant improvement in the ability to recover the transmitted symbols as evident in the symbol error probability. Above a SNR threshold of around $10 \mathrm{~dB}$ for the binary case and $12 \mathrm{~dB}$ for the multilevel case, the symbol error probability becomes negligible attributable to the fact that inverse filtering solution becomes nearly perfect after that threshold, which has been verified but not shown in Fig. 6 due to limitations of the log scale.

\section{Conclusions}

In this paper, we have presented an approach towards wideband CPM demodulation by extending the MFTESA approach developed by the authors. The characteristic features of the proposed MFT-ESA approach are (1) unlike the Viterbi algorithm whose complexity increases with the number of phase states induced by $m$ and $p$ as in Eq. 8, the complexity of the proposed approach is independent of the modulation index; (2) the proposed approach does not require prior knowledge of the carrier frequency and this parameter can be extracted from the IF estimates; and (3) the proposed approach accommodates large modulation indices and multilevel signaling making it conducive to the large bandwidth requirements proposed in the M-ary FSK system for satellite communications [15].

The proposed MFT-ESA approach was then applied to the demodulation of wideband CPM signals with partial response signaling, where memory is introduced into the estimated IF. Subsequent to the MFT-ESA demodulation stage, a recursive prediction approach based on MA signal modeling of the estimated IF, together with decision feedback equalization, was presented to address the problem 

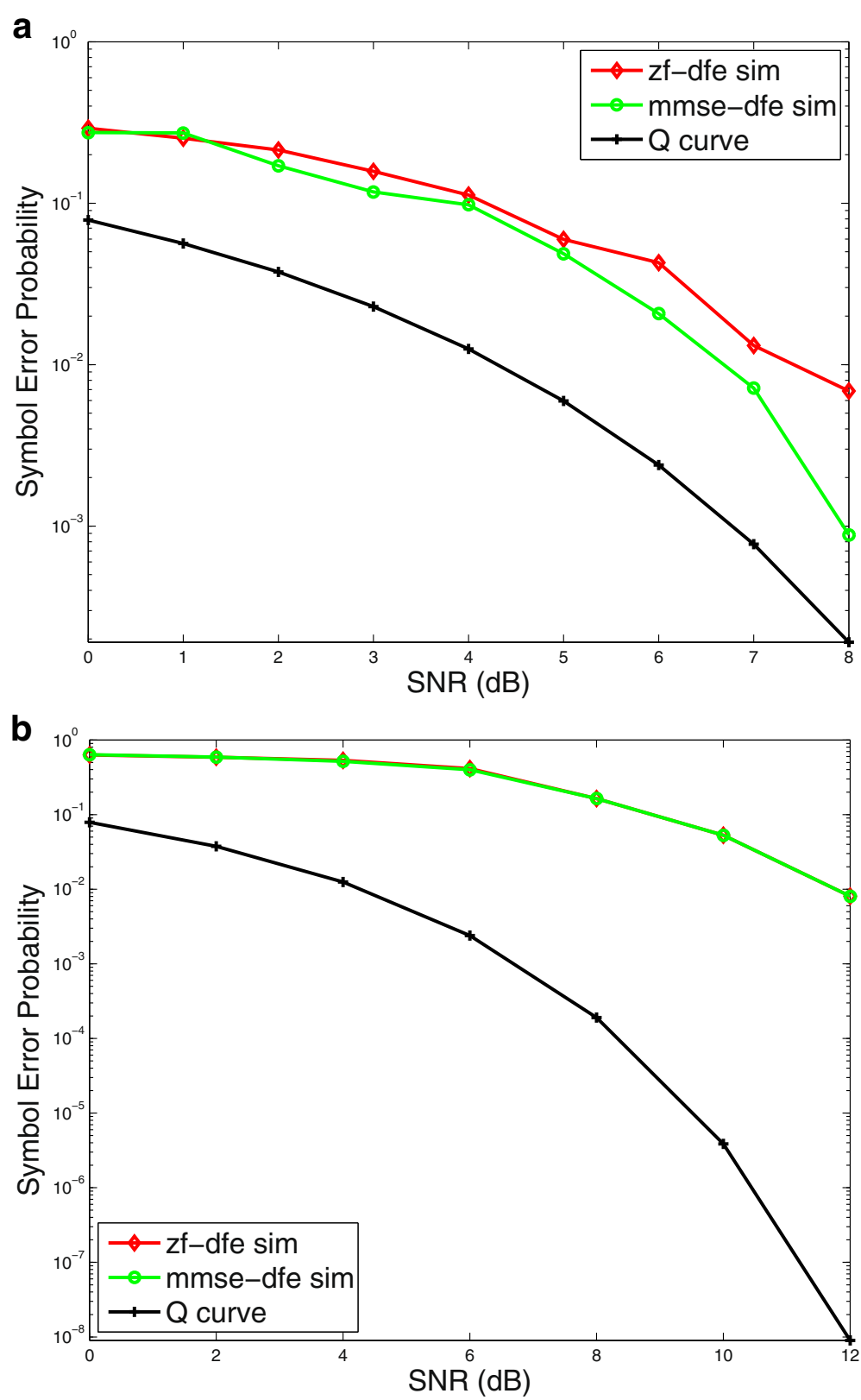

Fig. 6 Memory removal via decision feedback equalization: a symbol error probability of MFT-ESA approach for binary CPFSK with zero-forcing and MMSE decision feedback equalization to remove memory induced due to partial response signaling and $\mathbf{b}$ symbol error probability for multilevel CPFSK with zero-forcing and MMSE decision feedback equalization. In both cases, 3-REC CPM with parameters $T_{b}=1 \mathrm{~s}, f_{s}=50 \mathrm{~Hz}$, and $f_{c}=12 \mathrm{~Hz}$ was employed. The MFT conversion factor was $R=16$, and the modulation indices for the binary and multilevel cases were $h=97 / 21$ and $h=19 / 15$, respectively. For equalization purposes, 50 pilot symbols were used. Note that the SEP of $\mathbf{a}$ and $\mathbf{b}$ will drop to zero around 11 and $16 \mathrm{~dB}$, respectively, which are not shown due to limitations of the log scale

of removal of memory introduced by partial response signaling. Both the zero-forcing solution based on direct inversion of the memory channel, its corresponding decision feedback version and the MMSE-DFE solution to memory removal were investigated and shown to produce significant reduction in the symbol error probability over no equalization.

\section{Abbreviations}

AWGN: Additive white Gaussian noise; AM-FM: Amplitude-modulation frequency-modulation; BPSK: Binary phase-shift keying; CPM: Continuous phase modulation; CR/FD: Carrer-to-frequency-deviation ratio; CR/IB: Carrier-to-informaton-bandwidth ratio; DFE: Decision feedback equalization; ESA: Energy separation algorithm; FM: Frequency modulation; FSK: Frequency-shift keying; GMSK: Gaussian Minimum Shift Keying; HTDA: Hilbert transform demodulation algorithm; IA: Instantaneous amplitude; IF: Instantaneous frequency; ISI: Inter-symbol interference; MLSE: Maximum 
likelihood sequence estimation; MFT: Multirate frequency transformations; MMSE: Minimum mean-square error; RLS: Recursive least squares; SNR: Signal-to-noise ratio; ZF: Zero-forcing

\section{Funding}

This paper is based on research sponsored by the Air Force Research Labs (AFRL) under the agreement FA9453-16-1-0067.

\section{Authors' contributions}

Both authors contributed to design of the system and development of the algorithm. Both authors read and approved the submitted version of the manuscript.

\section{Competing interests}

The authors declare that they have no competing interests.

\section{Publisher's Note}

Springer Nature remains neutral with regard to jurisdictional claims in published maps and institutional affiliations.

Received: 8 November 2017 Accepted: 12 April 2018

Published online: 02 May 2018

\section{References}

1. MJ Gertsman, JH Lodge, Symbol-by-symbol MAP demodulation of CPM and PSK signals on Rayleigh flat-fading channels. IEEE Trans. Commun. 45(7), 788-799 (1997)

2. T Aulin, JB Anderson, C-EW Sundberg, Digital Phase Modulation. (Plenum, New York, 1986)

3. CE Sundberg, Continuous phase modulation. IEEE Commun. Mag. 24, 25-38 (1986)

4. S Li, N Zhang, S Lin, L Kong, A Katangur, MK Khan, M Ni, G Zhu, Joint admission control and resource allocation in edge computing for internet of things. IEEE Netw. 32(1), 72-79 (2018)

5. S Lin, L Kong, Q Gao, MK Khan, Z Zhong, X Jin, P Zeng, Advanced dynamic channel access strategy in spectrum sharing $5 \mathrm{G}$ systems. IEEE Wireless Commun. 24(5), 74-80 (2017)

6. A Perotti, A Tarable, S Benedetto, G Montorsi, Capacity-achieving CPM schemes. IEEE Trans. Inf. Theory. 56(4), 1521-1541 (2010)

7. W Xue, W Shang, SB Makarov, Y Xu, A phase trajectories optimization method for CPM signal based on Pan-function model. EURASIP J. Adv. Signal Process. 2016(1), 55 (2016)

8. JG Proakis, M Salehi, Digital Communications, Fifth Edition. (McGraw-Hill Publishing Company, New York, 1995), pp. 284-290

9. SS Abayesekara, in 2015 IEEE International Conference on Digital Signal Processing (DSP). Robust full response M-ary raised-cosine CPM receiver design via frequency estimation, (Singapore, 2015), pp. 935-939. https:// doi.org/10.1109/ICDSP.2015.7252014. http://ieeexplore.ieee.org/stamp/ stamp.jsp?tp $=$ \&arnumber $=7252014$ \&isnumber $=7251315$

10. B Santhanam, Generalized energy demodulation for large frequency deviations and wideband signals. IEEE Signal Process. Lett. 11(1), 341-344 (2004)

11. M Gupta, B Santhanam, in The Thrity-Seventh Asilomar Conference on Signals, Systems \& Computers, 2003. Adaptive linear predictive frequency tracking and CPM demodulation, vol. 1, (2003), pp. 202-206. https://doi. org/10.1109/ACSSC.2003.1291897. http://ieeexplore.iee.org/stamp/ stamp.jsp?tp=\&arnumber $=1291897$ \&isnumber $=28782$

12. W Liu, B Santhanam, in 2015 IEEE Signal Processing and Signal Processing Education Workshop (SP/SPE). Wideband-FM demodulation for large wideband to narrowband conversion factors via multirate frequency transformations, (Salt Lake City, 2015), pp. 7-12. https://doi.org/10.1109/ DSP-SPE.2015.7369519. http://ieeexplore.ieee.org/stamp/stamp.jsp?tp= \&arnumber $=7369519$ \&isnumber $=7369513$

13. P Maragos, JF Kaiser, TF Quatieri, Energy separation in signal modulations with application to speech analysis. IEEE Trans. Signal Process. 41(10), 3024-3051 (1993)

14. A Potamianos, P Maragos, A comparison of the energy operator and hilbert transform approach to signal and speech demodulation. Signal Process. 37(1), 95-120 (1994)

15. M Fitch, K Briggs, Gaussian multilevel FM for high-bandwidth satellite communications. University College of London (2004)
16. D Christopoulos, S Chatzinotas, G Zheng, J Grotz, B Ottersten, Linear and nonlinear techniques for multibeam joint processing in satellite communications. EURASIP J. Wireless Commun. Netw. 2012(1), 162 (2012)

17. SK Chronopoulos, C Koliopanos, CT Angelis, in Proceedings of the 3rd international conference on Mobile multimedia communications (MobiMedia '07). ICST (Institute for Computer Sciences, Social-Informatics and Telecommunications Engineering). Satellite multibeam signaling for multimedia services (ICST, Brussels, 2007), p. 4

18. W Liu, B Santhanam, Wideband image demodulation via bi-dimensional multirate frequency transformations. J. Optic. Soc. Am. A. 33, 1668-1678 (2016)

19. F Salzenstein, AO Boudraa, Multi-dimensional higher order differentia operators derived from the Teager-Kaiser energy-tracking function. Signal Process. 89(4), 623-640 (2009)

20. FW King, Hilbert Transforms, vol. 2. (Cambridge University Press Cambridge, Cambridge, 2009)

21. L Ljung, System Identification: Theory for the User, Second Edition. (Prentice Hall, New Jersey, 1999), pp. 370-372

22. SS Haykin, Adaptive Filter Theory, 4th Edition. (Prentice Hall Press, Upper Saddle River, 2005), pp. 436-440

23. R Fischer, J Huber, C Windpassinger, Signal processing in decision feedback equalization of intersymbol-interference and multiple-input/multiple-output channels: a unified view. Signal Process. 83(8), 1633-1642 (2003)

24. V Kavitha, V Sharma, Optimal MSE solution for a decision feedback equalizer. EURASIP J. Adv. Signal Process. 2012(1), 172 (2012)

\section{Submit your manuscript to a SpringerOpen ${ }^{\circ}$ journal and benefit from:}

- Convenient online submission

Rigorous peer review

- Open access: articles freely available online

- High visibility within the field

- Retaining the copyright to your article

Submit your next manuscript at $>$ springeropen.com 